\title{
Aplicabilidade e regulamentação sanitária da nanomedicina em grandes distúrbios do Sistema Nervoso Central (SNC)
}

\section{Applicability and sanitary regulation of nanomedicine in major Central Nervous System (CNS) disorders}

Antonio Claudio

Tedesco

Faculdade de Filosofia, Ciências e Letras

de Ribeirão Preto, Universidade de São Paulo (FFCLRP - USP), Ribeirão

Preto, SP, Brasil

E-mail atedesco@usp.br Andrielle Castilho-

Fernandes

Faculdade de Filosofia, Ciências e Letras de Ribeirão Preto, Universidade de São Paulo (FFCLRP - USP), Ribeirão

Preto, SP, Brasil

Tácila Gabriele Lopes Faculdade de Filosofia, Ciências e Letras de Ribeirão Preto, Universidade de São Paulo (FFCLRP - USP), Ribeirão Preto, SP, Brasil

\section{RESUMO}

Na nanomedicina, os nanocarreadores são geralmente biocompatíveis, biodegradáveis de rápida biodistribuição pelo organismo e podem ser utilizados para transportar drogas ou genes terapêuticos. Assim, novos sistemas de liberação de fármacos têm sido altamente explorados no tratamento de doenças do Sistema Nervoso Central (SNC), como Parkinson, Alzheimer e gliomas, visto que o SNC representa um grande desafio para as abordagens terapêuticas devido às barreiras hemato-encefálica (BHE) e sangue-líquido cefalorraquidiano (BSLCR). Desse modo, a comunidade científica juntamente com instituições governamentais e indústrias privadas vêm somando esforços para gerar novas formulações em escalas nanométricas com o intuito de alcançar uma abordagem terapêutica adequada, satisfatória e que esteja dentro dos princípios da vigilância sanitária para os acometimentos cerebrais. Este artigo visa sumarizar os conhecimentos sobre principais barreiras para entrega de fármacos ao SNC, nanomedicina, glioma, $\mathrm{Pa}$ rkinson, Alzheimer e vigilância sanitária.

PALAVRAS-CHAVE: Nanomedicina; Sistema Nervoso Central; Glioma; Parkinson, Alzheimer; vigilância sanitária

\section{ABSTRACT}

In nanomedicine the nanocarriers are generally biocompatible, biodegradable with rapid biodistribution in the body and can be used to carry drugs or therapeutic genes. Thus, new drug delivery systems have been heavily exploited in the treatment of CNS disorders such as Parkinson's, Alzheimer's and glioma, since the CNS is a major challenge for therapeutic approaches due to the blood-brain barrier (BBB) and blood-cerebrospinal fluid (BCSFB). Thus, the scientific community together with government and private industry has added efforts to generate new formulations in nanoscale in order to achieve an appropriate therapeutic approach, satisfying, and that is within the principles of health monitoring for cerebral affections. This article aims to summarize the knowledge about the main barriers to drug delivery to the CNS, Nanomedicine, Glioma, Parkinson's, Alzheimer's and sanitary surveillance.

KEYWORDS: Nanomedicine; Central Nervous System; Glioma; Parkinson's Alzheimer's; sanitary surveillance 


\section{Introdução}

Nos últimos anos, tem sido observado progresso significativo no campo da nanotecnologia, principalmente na área de ciências de materiais, estruturas supramoleculares, entrega de fármacos e terapia fotodinâmica. Em geral, os fármacos são desenvolvidos em nanopartículas (NP), termo aplicado a partículas inferiores ou iguais a $100 \mathrm{~nm}$ de tamanho. Entretanto, existe controvérsia que considera medicamentos e materiais com até $300 \mathrm{~nm}$ como estando rigorosamente dentro da escala que podemos chamar de nanométrica, tendo em vista a mudança de propriedades que apresentam. Na aplicação terapêutica da nanotecnologia, normalmente denominada nanomedicina, as NPs são, geralmente, biocompatíveis, biodegradáveis, de rápida biodistribuição pelo organismo e podem ser utilizadas para transportar fármacos ou genes terapêuticos, sendo chamadas de nanocarreadores. A maior vantagem no design desses sistemas é fazer com que sua especificidade pelo tecido alvo seja aumentada em 100 até 1.000 vezes, tornando-os alvos específicos. No passado, isso recebeu o nome de "bala mágica”, pois garantia que apenas as células ou organismos doentes recebessem esses ativos de interesse. Nesse período, o direcionamento era dado pela associação a anticorpos monoclonais, algo que ainda existe, mas de um custo muito elevado. Desde aquele período até os dias de hoje surgiram novas estratégias como a de desenhar sistemas de veiculação ou nanocarreadores com esse propósito.

Adicionalmente, é possível observar que ao longo das últimas três décadas houve um impulso crucial na geração de diversos nanocarreadores capazes de transportar medicamentos específicos para diversas doenças, como exemplo as que acometem o Sistema Nervoso Central (SNC). Esses nanocarreadores geralmente se enquadram nas seguintes categorias: lipossomas, NPs ligadas à albumina (tecnologia nab® patenteada), NPs poliméricas, dendrímeros e NPs metálicas ${ }^{1,2,3,4}$.

Vários nanocarreadores biodegradáveis contendo anticorpos monoclonais, peptídeos, proteínas, ácidos nucléicos, fatores de crescimento, entre outros, têm sido altamente explorados no tratamento de doenças do SNC (conhecidas como neurodegenerativas), como o Parkinson, Alzheimer, gliomas, dor e distúrbios psiquiátricos, pois cientistas acreditam que eles apresentam não só um profundo efeito contrário à progressão da doença, mas também restauram as células danificadas. Além disso, existe grande interesse por esses agentes bioterapêuticos, pois são bastante efetivos, potentes e reduzem os efeitos colaterais provocados pelos princípios ativos $2,5,6$.

Entretanto, são poucos os produtos biológicos desenvolvidos para o tratamento de desordens do SNC que obtiveram sucesso clínico. Esse fato ocorre pela carência de drogas com as seguintes propriedades associadas: boa solubilidade, estabilidade in vivo, forte penetração em todo o SNC e baixo custo de fabricação, limitando assim a entrada deles no mercado ${ }^{7,8}$. Desse modo, a comunidade científica juntamente com instituições governamentais e indústrias privadas vêm somando esforços para gerar novas formulações em escalas manométricas, com o intuito de alcançar uma abordagem terapêutica adequada e satisfatória para os acometimentos cerebrais.

\section{Principais barreiras para entrega de fármacos ao SNC}

O SNC representa um grande desafio para as abordagens terapêuticas. Adversamente às demais regiões do corpo, o SNC está particularmente bloqueado ao acesso de substâncias endógenas ou exógenas estranhas. Sendo assim, os maiores obstáculos encontrados pelos fármacos durante sua entrega ao SNC são as barreiras hemato-encefálica (BHE) e sangue-líquido cefalorraquidiano (BSLCR). Enquanto a BHE é constituída por vários tipos de células, incluindo células endoteliais, astrócitos, células da microglia e pericitos do SNC, a BSLCR é estabelecida por células epiteliais do plexo coroide.

A BHE faz interface entre o encéfalo e os vasos sanguíneos e é constituída por junções endoteliais que controlam a abertura e fechamento das junções célula-célula (junções apertadas e as junções endoteliais aderentes), que são os principais reguladores da permeabilidade celular, formando, portanto, uma barreira metabólica e física per $\mathrm{si}^{9,10}$. Assim, a BHE impede que macromoléculas atravessem as junções célula-célula que revestem o sistema vascular cerebral e que $98 \%$ dos medicamentos em potencial alcancem seus objetivos no $\mathrm{SNC}^{7}$.

A BSLCR é a segunda barreira que um fármaco administrado sistemicamente encontra antes de entrar no SNC. Semelhante à barreira endotelial, a BSLCR é encontrada no nível de junções apertadas únicas apical entre as células epiteliais do plexo coroide, as quais estão dispostas de uma maneira que limita e direciona o transporte de íons, nutrientes e células para o interior do líquido cefalorraquidiano (LCR) e a remoção de substâncias tóxicas para fora deste ${ }^{9,10}$.

A presença dessas barreiras no SNC tem grandes consequências clínicas, tornando ainda mais desafiadora a entrega de fármacos em regiões alvo intracranianas durante o tratamento de tumores cerebrais e de doenças neurodegenerativas, como Parkinson e Alzheimer. Atualmente, as terapias destinadas para as doenças que afetam o cérebro dependem muitas vezes de lesões vasculares e permeabilidade das barreiras BHE e BSLCR. Contrariamente aos tumores primários e secundários sistêmicos, que respondem aos agentes quimioterapêuticos administrados via sistema cardiovascular, as metástases intracranianas muitas vezes continuam a crescer ${ }^{11,12}$.

\section{Nanomedicina para o glioma}

O câncer consiste no aumento desordenado de células anormais, as quais apresentam tendência a ser agressivas e incontroláveis, o que resulta na formação de tumores ou neoplasias malignas ${ }^{13}$. Os tumores da glia, genericamente conhecidos como glioma, são as neoplasias mais comuns do SNC adulto e surgem a partir de astrócitos, oligodendrócitos e seus precursores. Esse tipo de câncer pode ser classificado em vários 
grupos de acordo com suas características histológicas. Os oligodendrogliomas e astrocitomas são os dois principais grupos que compõem o glioma ${ }^{14}$.

De acordo com a Organização Mundial da Saúde, os oligodendrogliomas são tumores de baixa malignidade e crescimento lento que ocorrem, principalmente, na substância branca do cérebro. Seu grau de malignidade, em geral, é de tumores de grau II, que pode aumentar nas recidivas e tornar-se oligodendroglioma anaplástico (grau III), que consiste em células tumorais com núcleos arredondados e homogêneos, com alta atividade mitótica, proliferação microvascular e necrose ${ }^{15}$. 0 astrocitoma é dividido segundo o grau de malignidade e agressividade, que em ordem crescente de anaplasia são denominados: astrocitoma pilocítico (grau I), astrocitoma difuso (grau II), astrocitoma anaplástico (grau III) e glioblastoma multiforme (grau IV) ${ }^{15,16}$.

O glioblastoma multiforme (GBM) é o tipo mais agressivo e frequente desses tumores, sendo comumente encontrado na região fronto temporal, podendo afetar os lobos parietais. Apresenta características distintas de atipia nuclear, rápido crescimento, proliferação microvascular, necrose, instabilidade genética e quimiorresistência ${ }^{17}$. Devido a tais características, GBMs apresentam prognóstico pouco favorável e a sobrevida média de quase todos os pacientes acometidos é de aproximadamente um ano ${ }^{16,18}$.

Os GBMs são subdivididos em primários e secundários, acometendo pessoas com diferentes idades e estabelecendo-se por meio de diferentes mecanismos. Histologicamente, ambos são indistinguíveis; contudo, diferem em relação a genética e comportamento biológico. O GBM primário é o mais comum (90\% dos casos), atingindo pessoas acima de 60 anos, desenvolve-se de forma rápida e sem evidências clínicas de lesões malignas precursoras. Já os GBMs secundários se estabelecem por meio da progressão de um glioma de baixo grau, como astocitoma difuso ou astrocitoma anaplásico, e atinge pessoas a partir de 45 anos.

Devido ao fato de que esses tumores surgem no SNC e afetam estruturas cerebrais, pacientes acometidos pelos gliomas comumente desenvolvem sintomas que incluem dores de cabeça, convulsões, papiloedema e vômitos, resultado do aumento da pressão intracraniana exercida pelo tumor e edema. Ataques epiléticos, alterações neurológicas com perda sensorial e/ou motora, problemas de memória e funções mentais são comuns nesses pacientes ${ }^{19}$.

A maioria dos gliomas é difícil de ser tratada devido ao crescimento infiltrativo das células tumorais e a sua resistência à terapia convencional. Mesmo as neoplasias benignas, constituídas por células anormais que não possuem a capacidade de se infiltrar no tecido adjacente do SNC, são de difícil tratamento devido a sua alta taxa proliferativa, tornando-se também um risco à vida do paciente. A abordagem terapêutica para gliomas malignos recém-diagnosticados permaneceu essencialmente inalterada durante décadas, consistindo em remoção cirúrgica da massa tumoral, tanto quanto possível, seguida de radioterapia concomitante com quimioterapia por temazolamida $(\mathrm{TMZ})^{20,21}$.
O GBM é altamente resistente ao tratamento quimioterápico, uma vez que as células anormais possuem uma alta capacidade de reparo no DNA, além de existirem várias células em diversos estágios do ciclo celular, e não somente na fase $\mathrm{G} 2 / \mathrm{M}$, e diversas áreas de hipóxia com células quimiorresistentes. Além disso, a barreira hematoencefálica (BHE) dificulta a entrega de drogas para o SNC22.

Nesse sentido, as nanopartículas poliméricas (NP) são uma boa opção tanto para veicular um fármaco quanto para incorporar um agente de contraste para o diagnóstico por imagem, como a ressonância magnética em tempo real. No estudo apresentado por Bernal e colaboradores ${ }^{23}$, foi gerada uma NP contendo óxido de ferro e carregada com o fármaco antiglioma, temazolamida (TMZ). Foi observada redução dos tumores cerebrais e maior sobrevida em modelo experimental de roedores.

Ling et al. ${ }^{24}$ desenvolveram uma NP superparamagnética baseada em PLGA (Ácido poli(d,l-lático-co-glicólico)) carreada de TMZ recoberto com polisorbato-80 (P80-TMZ/SPIO-NPs) e observaram um aumento significativo no uptake dessa formulação em modelo experimental in vitro, utilizando células C6 de glioma.

\section{Nanomedicina para a doença de Parkinson}

O Parkinson é uma doença crônica neurodegenerativa que afeta o SNC, comprometendo principalmente o sistema motor. Embora as causas dessa doença ainda não sejam completamente conhecidas, sabe-se que ela resulta da degeneração e morte de neurônios dopaminérgicos na substância cinzenta do cérebro. Esses neurônios são responsáveis por produzir a dopamina, um importante neurotransmissor que atua no controle dos movimentos ${ }^{25,26}$.

Segundo a Organização das Nações Unidas (ONU) existem pelo menos 4 milhões de pacientes no mundo que sofrem da doença de Parkinson. No Brasil, não se tem um número exato de pessoas portadoras da doença. Entretanto, o Ministério da Saúde estima que existam pelo menos 200 mil pessoas com a doença. A prevalência da doença de Parkinson é de cerca de 160 casos/100.000 habitantes no mundo inteiro e a taxa anual de incidência é de cerca de 20 novos casos/100.000 habitantes ${ }^{27}$. A prevalência e a incidência aumentam com a idade, e são maiores em pessoas com mais de 50 anos; em função do aumento da expectativa de vida da população, projeções para o ano de 2030 indicam que 9 milhões de pessoas no mundo inteiro sofrerão de doença de Parkinson ${ }^{28}$.

O diagnóstico da doença de Parkinson é clínico e baseia-se na presença dos sintomas motores mais comuns, que incluem tremor, rigidez muscular, constipação crônica, diminuição do esvaziamento da bexiga e alterações posturais. Outros sintomas não motores também são manifestados pelos pacientes, tais como o comprometimento da memória, depressão, alterações do sono e distúrbios do sistema nervoso autônomo ${ }^{26}$. 0 diagnóstico definitivo da doença é feito com base em achados de corpúsculos de Lewy na substância negra. Esses corpúsculos de 
Lewy são estruturas esféricas de 8 a $30 \mu \mathrm{m}$ de diâmetro, de coloração rosácea quando corados com hematoxilina e eosina ${ }^{29}$.

Embora ainda não exista cura para a doença de Parkinson, o tratamento farmacológico é baseado na melhora significativa dos sintomas, visando ao retardo do seu progresso, e pode envolver tanto o uso de medicamentos quanto de métodos não farmacológicos, como a fisioterapia ${ }^{27,30}$. Na fase inicial, a terapia pode ser iniciada com um inibidor da monoamina oxidase tipo B (MAO-B) ou com algum agonista dopaminérgico (bromocriptina, lisuride, pramipexol, pergolida e ropinirol). Para os casos mais avançados, recomenda-se a administração da levodopa ou L-Dopa ${ }^{30}$.

A L-Dopa (di-idroxifenilalanina) é o mais eficiente agente terapêutico para o tratamento da doença de Parkinson. Devido ao impedimento de o neurotransmissor dopamina atravessar a BHE, é usada a sua precursora, a L-Dopa, que sofre descarboxilação pela enzima dopa-descarboxilase e é convertida em dopamina ${ }^{27}$. Apesar de ser considerado o medicamento de referência para aliviar os sintomas, L-Dopa é indicada apenas nos casos mais avançados da doença, pois o seu uso prolongado pode causar reações adversas indesejáveis. Estas podem ser resultantes da conversão de grande parte, em torno de 95\%, da L-dopa em dopamina por enzimas presentes em outros órgãos que não o cérebro. Como a dopamina tem efeitos periféricos, ela pode causar náuseas e vômitos, taquicardia, midríase, agitação, insônia, alucinações e movimentos anormais involuntários conhecidos por discinesia ${ }^{27,31}$. Com o intuito de diminuir os efeitos indesejáveis, são coadministrados, com a L-dopa, inibidores da dopa-descarboxilase, como a carbidopa e benserazida, a fim de evitar a sua conversão antes de chegar ao cérebro ${ }^{30}$.

Uma forma de contornar o problema de entrega de fármacos é desenvolver um sistema de liberação capaz de carrear e reduzir a dose da dopamina ou direcionar a -L-dopa para o cérebro, evitando o uso de um inibidor da dopa-descarboxilase; dessa forma, as abordagens terapêuticas baseadas na nanomedicina têm se mostrado as mais promissoras. Como exemplo, o trabalho de Trapani e colaboradores ${ }^{32}$, que observaram maiores níveis de dopamina no órgão alvo - o cérebro - após a administração intravenosa de dopamina nanoparticulada em ratos, além de menor taxa citotóxica, quando comparada à administração de dopamina sozinha.

Nessa temática, o Centro de Nanotecnologia, Engenharia Tecidual e Fotoprocessos voltado a Saúde - Grupo de Fotobiologia e Fotomedicina FFCLRP-USP, Ribeirão Preto-SP, tem desenvolvido, em parceria com a Universidade de Brasília (UnB) e Santa Casa de São Paulo, sistemas altamente interessantes que têm se mostrado promissores no tratamento e interrupção da propagação da doença de Parkinson. Ainda em fase animal, os estudos usam sistemas de veiculação de fármacos produzidos e patenteados pela USP, em 2006, que permeiam a BHE aportando drogas, de forma mais eficiente que a convencional e evitando a propagação da patologia. Foi do sucesso desses estudos que surgiu a ideia de desenhar um sistema sinérgico, para o tratamento do glioma, em que a associação de um quimioterápico e um fármaco fotoativável permitiria que o tumor reduzisse de tamanho e, consequentemente, permitiria a realização de uma cirurgia com uma margem muito menor, resultando, por fim, em comprometimento menor da zona tumoral, de modo similar ao que é usado hoje, clinicamente, no tratamento do câncer de pele, com fotoativação luminosa, visível, de fármacos naturais que ajudam a erradicar os resquícios de células doentes. Assim, portanto, a nanotecnologia, fotoprocesso e engenharia de tecidos trabalhando em conjunto em benefício da saúde humana.

$\mathrm{Na}$ área da terapia gênica para o Parkinson, as nanopartículas têm sido utilizadas como vetores de entrega de genes terapêuticos em substituição aos vírus (retrovírus, lentivírus, adenovírus...), tendo como objetivo anular o potencial mutagênico e a resposta imunológica. Utilizando essa abordagem terapêutica, Huang e colaboradores ${ }^{33}$ demonstraram um aumento dos níveis de dopamina no cérebro de ratos, além de uma melhora significativa da atividade motora e redução da perda neuronal.

\section{Nanomedicina para a doença de Alzheimer}

Dentre as doenças neurodegenerativas, o Alzheimer é a mais frequente em indivíduos com idade superior a 65 anos, sendo o fator genético considerado como preponderante na etiopatogenia da doença. O Alzheimer é uma epidemia, com estimativa de 33,9 milhões de pessoas no mundo com a doença, cujas manifestações cognitivas e neuropsiquiátricas resultam em deficiência progressiva e eventual incapacitação ${ }^{34,35}$. Normalmente, o primeiro sintoma se dá pela deficiência da memória recente, seguida por dificuldades de atenção e verbalização, enquanto as lembranças antigas são preservadas nos estágios iniciais da doença. As demais funções cognitivas deterioram progressivamente e são acompanhadas por distúrbios comportamentais, como agressividade, apatia, alucinações, hiperatividade, irritabilidade e depressão com o avanço da doença ${ }^{36,37}$.

A doença de Alzheimer caracteriza-se, histopatologicamente, pela maciça perda sináptica e pela morte neuronal no córtex cerebral, hipocampo, córtex entorrinal e estriado ventral, sendo essas regiões cerebrais as responsáveis pelas funções cognitivas. São observados, ainda, depósitos fibrilares amiloidais, placas senis e novelos neurofibrilares (NFT) nas paredes dos vasos sanguíneos do parênquima dos portadores do Alzheimer, além da perda neuronal e sináptica, ativação da glia e inflamação.

Apesar de ser bastante estudada, ainda pouco se sabe sobre a patogênese da doença de Alzheimer. Logo, foram desenvolvidas duas hipóteses para o início da neurodegeneração do Alzheimer: a da cascata amiloidal, que se inicia com a clivagem proteolítica da proteína precursora amilóide (APP) e resulta na produção, agregação e deposição da substância B-amilóide (AB) e placas senis; e a da disfunção do sistema colinérgico, onde ocorre degeneração dos neurônios colinérgicos e redução dos marcadores colinérgicos, sendo que a colina acetiltransferase e a acetilcolinesterase apresentam atividades reduzidas no córtex cerebral de pacientes portadores da doença de Alzheimer ${ }^{38,39}$.

Postula-se que a utilização de biomarcadores apropriados poderia permitir a identificação de alterações patológicas características do Alzheimer antes que se desenvolva o dano 
neuronal ou início da manifestação dos sintomas, admitindo-se, também, que o tratamento deve ser iniciado tão cedo quanto possível. Atualmente, não existe cura para o Alzheimer e os tratamentos existentes são paliativos que visam retardar o avanço da doença e minimizar os sintomas. Os medicamentos disponíveis para o tratamento são o donepezil, galantamina, rivastigmina e memantina, que agem evitando a queda dos níveis de acetilcolina, neurotransmissor presente nas sinapses, ou a formação de placas $A B^{40,41}$. Entretanto, estima-se que mais de 400 novos medicamentos estão sendo investigados em cerca de 1.100 ensaios clínicos realizados em humanos (ClinicalTrials.gov, 2013), e várias outras substâncias estão sendo testadas em modelo animal da doença de Alzheimer. Essas novas drogas têm como objetivo atingir profundamente as regiões afetadas do cérebro e para isso devem atravessar efetivamente a $\mathrm{BHE}^{41,42}$.

Dentre os nanofármacos relacionados à cascata amiloidal, destacam-se os anticorpos nanoencapsulados anti-AB por terem sua biodisponibilidade elevada e permanecerem na circulação por um tempo maior, como, por exemplo, a Curcumina, que inibe a formação da placa $A B$ e reduz os níveis de amiloide in vivo ${ }^{43}$. Mulik e colaboradores ${ }^{44}$ observaram que a nanopartícula de curcumina quando envolta por apo-Lipoproteína $E$ (ApoE), um ligante para o receptor expresso pela $\mathrm{BHE}$, permitiu uma redução de $40 \%$ da citotoxicidade relacionada a $A B$. 0 grupo de Matthew e colaboradores ${ }^{45}$ observou ainda que a curcumina nanopartículada com o peptídeo Tet-1, que tem afinidade para os neurônios, contorna a BHE e atinge os neurônios alvos.

Com relação à disfunção do sistema colinérgico, os nanotubos de carbono foram utilizados com o intuito de superar a baixa meia-vida da acetilconlina e facilitar sua penetração pela $\mathrm{BHE}^{46}$. Nanopartículas de rivastigmina quando revestidas com polysorbato- 80 absorvem a ApoE presente na corrente sanguínea; dessa forma, facilitam sua penetração pela BHE e permitem que haja uma melhora na aprendizagem e uma menor perda de memória. Essa mesma formulação, comparada a nanopartículas de rivastigmina não revestida com polysorbato-80 quando testadas em camundongos, apresentou uma penetração 12 vezes maior do que a não revestida ${ }^{47}$.

\section{Nanomedicina sob o olhar da vigilância sanitária}

Segundo dados prospectivos, a nanomedicina está em segundo lugar nos segmentos industriais impactados pela nanotecnologia no Brasil, atrás apenas do setor de material eletrônico e comunicações ${ }^{48}$. Nesse sentido, as pesquisas no campo da nanomedicina têm sido apontadas como uma das áreas de grande potencial para atender não somente às necessidades encontradas no Sistema Único de Saúde (SUS), mas também atender aos objetivos do milênio das Organizações das Nações Unidas (ONU), entre elas o mapeamento e diagnóstico de doenças, sistema para liberação de fármacos e monitoramento da saúde ${ }^{49}$.

No âmbito do sistema de liberação de fármacos é possível perceber que atualmente existe um déficit de abordagens te- rapêuticas eficazes para as doenças que acometem o SNC. Fato esse que vai ao encontro das necessidades encontradas pelo SUS, sugerindo, dessa forma, que haja um direcionamento nas linhas de pesquisas, inserido-as na proposta de prioridades de pesquisa em saúde pública ${ }^{50}$.

Diante disso, a construção de uma política nacional de pesquisa em saúde, incluindo acometimentos do SNC, deve passar por implementação de um programa estratégico com prioridades nas abordagens terapêuticas e agregar consistentemente em seu escopo os princípios da vigilância sanitária. Embora os objetivos da Agência Nacional de Vigilância Sanitária (Anvisa) sejam a regulamentação de produtos e serviços, é fundamental a existência do diálogo com a área científica e condecorá-la como uma vertente da saúde coletiva ${ }^{51,52,53}$.

Com o intuito de traçar diretrizes de como a pesquisa científica relacionada à nanotecnologia poderá desenvolver uma cultura de inovação e avanços para fortalecer a saúde pública e normatizar a nanotecnologia no Brasil, a Anvisa promoveu recentemente em Brasília-DF o "Seminário de Inovação Tecnológica em Saúde: Desafios para a Regulação Sanitária”, momento no qual foram discutidas as principais questões relativas à nanotecnologia aplicada na área da saúde, abordando principalmente os aspectos conceituais, aplicações, benefícios, potenciais riscos, desafios e os modelos regulatórios mais eficazes na atualidade ${ }^{54}$. Dentre os grupos científicos que estão na fronteira do desenvolvimento de produtos para a saúde e que participaram do evento estava o Centro de Nanotecnologia, Engenharia Tecidual e Fotoprocessos voltado a Saúde - Grupo de Fotobiologia e Fotomedicina FFCLRP-USP, Ribeirão Preto-SP, que detém uma forte linha de pesquisa voltada para as inovações tecnológicas na área de doenças neurodegenerativas: Parkinson, Alzheimer e Glioma, lembrando que esse grupo visa à pesquisa de interesse social para apoio ao desenvolvimento institucional do SUS, priorizando estudos de avaliação e incorporação de novas tecnologias, capacitação de recursos humanos, aplicabilidade para o sistema público em saúde e o desenvolvimento de técnicas em serviços de saúde, visando sempre atender ao decreto federal $n^{\circ} 5895$.

\section{Conclusão}

Uma das esperanças da nanomedicina no tratamento das doenças neurodegenerativas, como o Parkinson, Alzheimer e glioma, reside na capacidade das nanopartículas em ultrapassar a chamada BHE e possibilitar, por exemplo, uma terapia gênica no nível do sistema nervoso central. Além disso, podemos citar ainda como potenciais benefícios a administração não invasiva e otimização da biodistribuição do fármaco, redução dos efeitos colaterais e melhores resultados terapêuticos, podendo assim aumentar as chances de sobrevida em pacientes com tumores cerebrais.

A transposição do laboratório de pesquisa para a clínica é o principal desafio na aplicação de sistemas de entrega alvo, já que para ser autorizado no mercado a Anvisa exige a realização de um processo longo e elaborado de três fases pré-clínicas e três fases de ensaios clínicos, o que leva vários anos 
para ser concluído. Atualmente, apenas alguns medicamentos com tecnologia nano foram aprovados pela Anvisa e estão comercialmente disponíveis, incluindo doxorrubicina carregado por lipossoma para tratamento de câncer de ovário e mama metastático. É possível observar que o reduzido número de fármacos nanoestruturados e aprovados, para utilização clínica, está diretamente relacionado à escassez de leis específicas que avaliem a toxicidade desses sistemas tanto para a saúde humana quanto para o meio ambiente, demonstrando, assim, que existe urgência na consolidação de uma regulamentação sólida para esses medicamentos nanoparticulados. De modo geral, progressos substanciais estão acontecendo no campo da nanomedicina e diversos grupos de pesquisa, no mundo todo, estão concentrando esforços para desenvolver práticas clínicas eficazes e seguras que utilizam sistemas de veiculação em escala nano em prol de acometimentos do SNC.

\section{Referências}

1. Bhattacharya R, Mukherjee P. Biological properties of "naked" metal nanoparticles. Adv Drug Deliv Rev. 2008;60(11):1289-306.

2. Mukerjee A, Ranjan AP, Vishwanath JK. Combinatorial nanoparticles for cancer diagnosis and therapy. Curr Med Chem. 2012;19(22):3714-21.

3. Shah L, Yadav S, Amiji M. Nanotechnology for CNS Delivery of Bio-Therapeutic Agents. Drug Deliv Transl Res. 2013;3(4):336-51.

4. Vishnu P, Roy V. Safety and Efficacy of nab-Paclitaxel in the Treatment of Patients with Breast Cancer. Breast Cancer (Auckl). 2011;5:53-65.

5. McGonigle P. Peptide therapeutics for CNS indications. Biochem Pharmacol. 2012;83(5):559-66.

6. Andrieux K, Couvreur P. Nanomedicine as a promising approach for the treatment and diagnosis of brain diseases:The example of Alzheimer's disease. Ann Pharm Fr. 2013;71(4):225-33.

7. Rajadhyaksha M, Boyden T, Liras J, El-Kattan A, Brodfuehrer J. Current Advances in Delivery of Biotherapeutics Across the Blood-Brain Barrier. Curr Drug Discov Technol. 2011;8(2):87-101.

8. Khanbabaie R, Jahanshahi $M$. Revolutionary impact of nanodrug delivery on neuroscience. Curr Neuropharmacol. 2012;10(4):370-92.

9. Engelhardt B, Sorokin L.The blood-brain and the blood-cerebrospinal fluid barriers: function and dysfunction. Semin Immunopathol. 2009;31(4):497-511.

10. Stamatovic SM, Keep RF, Andjelkovic AV. Brain endothelial cell-cell junctions: how to "open" the blood brain barrier. Curr Neuropharmacol. 2008;6(3):179-92.

11. Misra A, Ganesh S, Shahiwala A, Shah SP. Drug delivery to the central nervous system: a review. J Pharm Pharm Sci. 2003;6(2):252-73.

12. Re F, Gregori M, Masserini M. Nanotechnology for neurodegenerative disorders. Nanomedicine. 2012;8 Suppl 1:S51-8.
13. De Mejia EG, Bradford T, Hasler C. The anticarcinogenic potential of soybean lectin and lunasin. Nutr Rev. 2003;61(7):239-46.

14. Ohgaki H. Genetic pathways to glioblastomas. Neuropathology. 2005;25(1):1-7.

15. Kleihues P, Cavernee WK eds. Pathology and genetics tumours of the nervous system. In: World Heath Organization Classification of Tumours, v.1. IARC press: Lyon; 2000.

16. Louis DN, Ohgaki H, Wiestler OD, Cavenee WK, Burger PC, Jouvet A, Scheithauer BW, Kleihues P. The 2007 WHO classification of tumours of the central nervous system. Acta Neuropathol. 2007;114(2):97-109.

17. Furnari FB, Fenton T, Bachoo RM, Mukasa A, Stommel JM, Stegh A, Hahn WC, Ligon KL, Louis DN, Brennan C, Chin L, DePinho RA, Cavenee WK. Malignant astrocytic glioma: genetics, biology, and paths to treatment. Genes Dev. 2007;21(21):2683-710.

18. Collins VP. Brain tumours: classification and genes. J Neurol Neurosurg Psychiatry. 2004;75 Suppl 2:ii2-11.

19. Behin A, Hoang-Xuan K, Carpentier AF, Delattre JY. Primary brain tumours in adults. Lancet. 2003;361(9354):323-31.

20. Stupp R, Mason WP, van den Bent MJ, Weller M, Fisher B, Taphoorn MJ, Belanger K, Brandes AA, Marosi C, Bogdahn U, Curschmann J, Janzer RC, Ludwin SK, Gorlia T, Allgeier A, Lacombe D, Cairncross JG, Eisenhauer E, Mirimanoff RO; European Organisation for Research and Treatment of Cancer Brain Tumor and Radiotherapy Groups; National Cancer Institute of Canada Clinical Trials Group. Radiotherapy plus concomitant and adjuvant temozolomide for glioblastoma. N Engl J Med. 2005;352(10):987-96.

21. Kanu OO, Mehta A, Di C, Lin N, Bortoff K, Bigner DD, Yan H, Adamson DC. Glioblastoma multiforme: a review of therapeutic targets. Expert Opin Ther Targets. 2009;13(6):701-18.

22. Selbo PK, Weyergang A, Eng MS, Bostad M, Mælandsmo GM, Høgset A, Berg K. Strongly amphiphilic photosensitizers are not substrates of the cancer stem cell marker ABCG2 and provides specific and efficient light-triggered drug delivery of an EGFR-targeted cytotoxic drug. J Control Release. 2012;159(2):197-203.

23. Bernal GM, Lariviere MJ, Mansour N, Pytel P, Cahill KE, Voce DJ, Kang S, Spretz R, Welp U, Noriega SE, Nunez L, Larsen GF, Weichselbaum RR, Yamini B. Convection-enhanced delivery and in vivo imaging of polymeric nanoparticles for the treatment of malignant glioma. Nanomedicine. 2013. pii: S15499634(13)00343-2.

24. Ling Y, Wei K, Zou F, Zhong S. Temozolomide loaded PLGA-based superparamagnetic nanoparticles for magnetic resonance imaging and treatment of malignant glioma. Int J Pharm. 2012;430(1-2):266-75.

25. Ren JP, Zhao YW, Sun XJ. Toxic influence of chronic oral administration of paraquat on nigrostriatal dopaminergic neurons in C57BL/6 mice. Chin Med J (Engl). 2009;122(19):2366-71.

26. Arbouw ME, Guchelaar HJ, Egberts TC. Novel insights in pharmacogenetics of drug response in Parkinson's disease. Pharmacogenomics. 2010;11(2):127-9. 
27. Pahwa R, Lyons KE. Early diagnosis of Parkinson's disease: recommendations from diagnostic clinical guidelines. Am J Manag Care. 2010;16:S94-S9.

28. Dorsey ER, Constantinescu R, Thompson JP, Biglan KM, Holloway RG, Kieburtz K, Marshall FJ, Ravina BM, Schifitto G, Siderowf A, Tanner CM. Projected number of people with Parkinson disease in the most populous nations, 2005 through 2030. Neurology. 2007;68(5):384-6.

29. Olanow CW, Perl DP, DeMartino GN, MCNaught KSP. Lewy-body formation is an aggresome-related process: a hypothesis. Lancet Neurol. 2004;3:496-503.

30. Stewart DA. NICE guideline for Parkinson's disease. Age and Ageing. 2007;36:240-2.

31. Manjunath S, Sakhare PM. Adenosine and adenosine receptors: newer therapeutic perspective. Indian J Pharmacol. 2009;41(3):97-105.

32. Trapani A, De Giglio E, Cafagna D, Denora N, Agrimi G, Cassano T, Gaetani S, Cuomo V, Trapani G. Characterization and evaluation of chitosan nanoparticles for dopamine brain delivery. Int J Pharm. 2011;419(1-2):296-307.

33. Huang R, Ke W, Liu Y, Wu D, Feng L, Jiang C, Pei Y. Gene therapy using lactoferrin-modified nanoparticles in a rotenone-induced chronic Parkinson model. J Neurol Sci. 2010;290(1-2):123-30.

34. Lindeboom J, Weinstein H. Neuropsychology of cognitive ageing, minimal cognitive impairment, Alzheimer's disease, and vascular cognitive impairment. Eur J Pharmacol. 2004;490(1-3):83-6.

35. Banks W A. Drug delivery to the brain in Alzheimer's disease: consideration of the blood-brain barrier. Adv Drug Deliv Rev. 2012;64(7):629-39.

36. Husain MM, Garrett RK. Clinical diagnosis and management of Alzheimer's disease. Neuroimaging Clin N Am. 2005;15(4):767-77.

37. Lazarczyk MJ, Hof PR, Bouras C, Giannakopoulos P. Preclinical Alzheimer disease: identification of cases at risk among cognitively intact older individuals. BMC Med. 2012;10:127.

38. Auld DS, Kornecook TJ, Bastianetto S, Quirion R. Alzheimer's disease and the basal forebrain cholinergic system: relations to beta-amyloid peptides, cognition and treatment strategies. Prog Neurobiol. 2002;68(3):209-45.

39. Lynch MA. The impact of neuroimmune changes on development of amyloid pathology; relevance to Alzheimer's disease. Immunology. 2013. [Epub ahead of print]

40. Agência Nacional de Vigilância Sanitária (BR). Alerta terapêutico $n^{\circ}$ 04/02 - Tartarato de Rivastigmina-EXELON® ATC: N06D A03 [Internet]. Brasília: ANVISA; 2002. [acesso em 14 jun. 2013]. Disponível em: http://s.anvisa.gov.br/ $\mathrm{wps} / \mathrm{s} / \mathrm{r} / \mathrm{bjyj}$

41. Di Stefano A, lannitelli A, Laserra S, Sozio P. Drug delivery strategies for Alzheimer's disease treatment. Expert Opin Drug Deliv. 2012;8(5):581-603.
42. Becker RE, Greig NH, Giacobini E. Why do so many drugs for Alzheimer's disease fail in development? Time for new methods and new practices? J Alzheimers Dis. 2008;15(2):303-25.

43. Yang F, Lim GP, Begum AN, Ubeda OJ, Simmons MR, Ambegaokar SS, Chen PP, Kayed R, Glabe CG, Frautschy SA, Cole GM. Curcumin inhibits formation of amyloid beta oligomers and fibrils, binds plaques, and reduces amyloid in vivo. J Biol Chem. 2005;280(7):5892-901.

44. Mulik RS, Mönkkönen J, Juvonen RO, Mahadik KR, Paradkar AR. ApoE3 mediated poly(butyl) cyanoacrylate nanoparticles containing curcumin: study of enhanced activity of curcumin against beta amyloid induced cytotoxicity using in vitro cell culture model. Mol Pharm. 2010;7(3):815-25.

45. Mathew A, Fukuda T, Nagaoka Y, Hasumura T, Morimoto H, Yoshida Y, Maekawa T, Venugopal K, Kumar DS. Curcumin loaded-PLGA nanoparticles conjugated with Tet-1 peptide for potential use in Alzheimer's disease. PLoS One. 2012;7(3):e32616.

46. Yang Z, Zhang Y, Yang Y, Sun L, Han D, Li H, Wang C. Pharmacological and toxicological target organelles and safe use of single-walled carbon nanotubes as drug carriers in treating Alzheimer disease. Nanomedicine. 2010;6(3):427-41.

47. Joshi SA, Chavhan SS, Sawant KK. Rivastigmine-loaded PLGA and PBCA nanoparticles: preparation, optimization, characterization, in vitro and pharmacodynamic studies. Eur J Pharm Biopharm. 2010;76(2):189-99.

48. Agência Brasileira de Desenvolvimento Industrial. Estudo prospectivo de nanotecnologia. Brasília: ABDI; 2011.

49. Salamanca-Buentello F, Persad DL, Court EB, Martin DK, Daar AS, Singer PA. Nanotechnology and the developing world. PLoS Med. 2005;2(5):e97.

50. Brasil, Ministério da Saúde, Secretaria de Ciência, Tecnologia e Insumos Estratégicos. Departamento de Ciência e Tecnologia. Agenda Nacional de Prioridades de Pesquisa em Saúde. Brasília: Ed. Ministério da Saúde; 2008. (Série B. Textos Básicos em Saúde).

51. Agência Nacional de Vigilância Sanitária (BR). Plano Estratégico de Pesquisa em Vigilância Sanitária. Brasília: ANVISA; 2007.

52. Agência Nacional de Vigilância Sanitária (BR). Agenda Nacional de Prioridades de Pesquisa em Vigilância Sanitária. Brasília: Núcleo de Educação, Pesquisa e Conhecimento NEPEC/ANVISA; 2011.

53. Piovesan MF, Labra ME. Institutional change and political decision-making in the creation of the Brazilian National Health Surveillance Agency. Cad Saude Publica. 2007;23(6):1373-82.

54. Agência Nacional de Vigilância Sanitária (BR). Seminário de Inovação Tecnológica em Saúde: Desafios para a Regulação Sanitária; 26-28 jun. 2013. Brasília: ANVISA; 2013. 